\title{
Energy Heterogeneous Wireless Sensor Network Clustering Mechanism in Farmland
}

\author{
Dayong Liu ${ }^{1}$, Chunjiang $\mathrm{Zhao}^{2,3, *}$, Huarui $\mathrm{Wu}^{2,3}$ and Feifei $\mathrm{Li}^{2,3}$ \\ ${ }^{1}$ College of Computer Science and Technology, Beijing University of Technology, Beijing 100022, China \\ ${ }^{2}$ Beijing Research Center for Information Technology in Agriculture, Beijing Academy of Agriculture and Forestry \\ Sciences, Beijing 100097, China \\ ${ }^{3}$ National Engineering Research Center for Information Technology in Agriculture, Beijing 100097, China
}

\begin{abstract}
Solar-powered nodes employed in farmland wireless sensor network have energy advantages but at the same time have the problem of high cost. Wireless sensor network employs both solar-powered nodes and battery powered nodes which can reduce cost and prolong network lifetime. This paper presents an algorithm based on LEACH to study the clustering process of farmland environment monitoring sensor network which contains both solar-powered nodes and battery-powered nodes. This algorithm classifies nodes into three types based on the nodes characteristics first and the nit selects cluster nodes based on the comparison of the number of cluster head nodes and the solar powered nodes. The algorithm in this paper gives priority to utilize the solar-powered battery by two elections in one round. Simulation result shows that this algorithm can improve the utilization of solar energy, decrease node death and finally prolong the network lifecycle.
\end{abstract}

Keywords: Wireless sensor network, energy heterogeneous, solar-powered, collaborative work, hierarchical structure.

\section{INTRODUCTION}

Sensor technology and intelligent information processing are important means to ensure effective and timely access to the agricultural information. Large scale arrangement of sensor nodes in farmland can help the manager to get the environment parameters and provide them with reliable information for farmland management. At the same time, farmland monitoring is a long time task because of the growth period of the crops and the remote agricultural production environment is away from the power supply facilities, which makes the sensor nodes unable to get sustained and unlimited power supply from the electricity grid.

Now the most common mode of power for sensor nodes is battery power. But battery-driven sensors will run out of power eventually, the use of renewable energy sources such as solar power may extend the lifetime of a sensor network. There is a nabundance of solar energy resources in agricultural environment. Solar energy is able to meet the energy requirements of sensor nodes. However, the solar powered nodes have higher cost and the energy supply is affected by the weather condition. Nodes can't get enough power in a continuous rainy weather. Wireless sensor network that employs both solar powered and battery powered nodes can solve both energy and cost problems.
Because of the limited number of solar powered nodes in the network, it is necessary to establish a mechanism for collaborative work among nodes of different type. By improving the solar energy nodes' energy consumption and at the same time reducing battery powered nodes' energy consumption, this mechanism improves the utilization of solar energy and at last achieves the purpose of prolonging the network lifetime.

This paper constructs a mechanism to select cluster head nodes and to achieve collaborative work between the nodes with different power supplies: in the case that the solar powered nodes can get enough energy, the network increase the utilization of solar energy and reduces the energy consumption of battery-powered nodes; in the case that the solar powered nodes can't get enough energy, the network balances the energy consumption of different nodes to prevent excessive energy consumption.

\section{RELATED WORKS}

Wireless sensor network is divided into two types: hierarchical structure network and flat structure network. A hierarchical network has the advantages of saving energy and improving network performance [1-3].

Nodesare divided into different layers in different clusters in the hierarchical network. A cluster consists of some adjacent nodes. Each cluster has a cluster head. Clusters communicate either directly or via a gateway. The gateway can be other cluster head. The connection between gateways constitute the upper layer — the backbone layer. The com- 


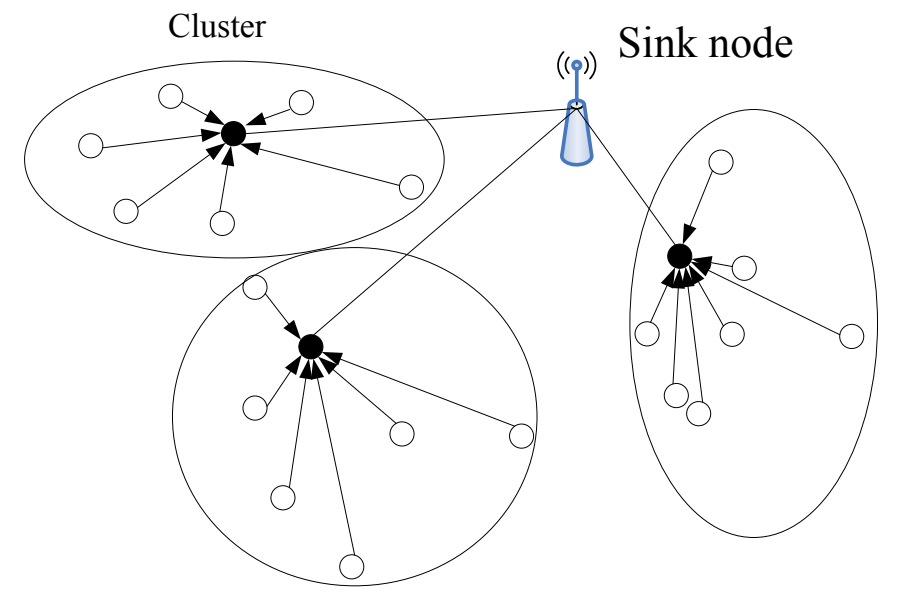

\section{- Cluster head node}

Fig. (1). Hierarchical structure diagram.

munication among clusters is conducted via the backbone network [4-6]. In the hierarchical structure network, nodes in different layer undertake different amount of data transfer work, so they have different energy consumptions. The reasonable cluster head selection policy and nodes collaborative work would bring energy balance and the efficient use of energy. The Fig. (1) shows the hierarchical structure diagram.

Clustering of network reduces long-distance wireless communication, consequently reduces energy consumption and prolongs network lifecycle. By cluster head rotation, network achieves energy consumption balance among nodes. Several works and studies have been performed regarding energy-efficient clustering in wireless sensor networks. The typical and commonly used algorithm is LEACH algorithm. LEACH [7-8] (Low Energy Adaptive Clustering Hierarchy) is a typical hierarchical clustering routing protocol. But the LEACH algorithm uses random strategy to select cluster head as backbone nodes. The random strategy does not take the nodes energy condition into account, which would not be conductive to the effective use of energy specifically in energy heterogeneous networks.

Researchers made some improvements to LEACH to achieve low energy consumption and energy balance [9-14]. YANG Yong-jian et al. [15] optimized the mechanism of cluster head election by estimating the residual energy of nodes and proposed the sleeping scheduling model based on" game of life" and communication of multi-hops which used its neighbor nodes as forwarding nodes to prolong network life cycle and increase the amount of data transmission. CHEN Xiaojuan et al. [16] proposed an improved and energy-efficient routing protocol to balance the network load efficiently, improve the energy utilization and prolong the network lifetime. It first cuts the wireless senor network into more than one sub domains by PSO and then selects cluster heads within the factor of node residual energy. CHEN Zhen-yan et al. [17] presents an improved LEACH algorithm based on mobile cluster heads. As clusters lose energy or become unbalanced after a period of network operation, mo- bile sensors are added to clusters and promoted to cluster head in order to prolong the network survival time.

These researches prolonged network lifecycle by reduce energy consumption and achieve energy balance. In order toachieve the goal of energy balance, these existing algorithms select cluster head nodes based on the nodes residual energy. These strategies do not apply to networks which employ both battery powered nodes and solar powered nodes. That is because the solar powered nodes obtain the energy from the environment and the residual energy changes vary differently depends on power supply types. The mobile clusterheads strategy does not apply to networks either because of high costs and complex environment in farmland.

\section{PROBLEM DESCRIPTION}

Nodes with different power supply type have different energy changing characteristics. In the network which employs both solar powered nodes and battery powered nodes, it is necessary to consider the characteristics of different node types in the hierarchical network formation process: solar-powered nodes get energy from the environment and the energy acquisition is dependent on the weather condition and the circadian cycle. That means a solar powered node has different energy acquisition model in different weather conditions and in different time periods: the node gets the solar energy in the sunny weather during the day but not in the cloudy or rainy weather or during the night. The energy of battery powered nodes monotonically decreases all the time.

Because of factors mentioned above, cluster head selection and clustering in this network becomes complicated. The cluster head nodes consume more energy than the cluster member nodes, so if the selected solar powered nodes as cluster head nodes than they can improve the utilization of solar energy and reduce the energy consumptions of battery in the sunny weather. But in the consecutive rainy days, using a solar powered node with low residual energy as cluster head node may lead to node death because of rapid depletion 
of energy. The data of the entire cluster could not be uploaded if the cluster head nodes energy depletes. This condition results in a large area of monitoring coverage hole.

So we should establish a mechanism of energy heterogeneous nodes in wireless sensor network to ensure both network stability and network lifetime. This paper studies how to establish collaborative work mechanism of energy heterogeneous nodes in wireless sensor network. The mechanism improves the solar energy utilization in good weather with adequate light and concerns about the energy balance between the two different types of nodes to avoid some nodes being depleted of energy in bad weather.

\section{CLUSTERINGALGORITHMBASED ON NODES CLASSIFYING}

\subsection{Network Model and Parameter Definitions}

The wireless sensor network in this paper has the following characteristics:

1)All nodes are static nodes;

2)All the nodes are randomly distributed in the monitoring environment, and the node density is large enough;

3)The solar-powered nodes can get enough energy in good weather conditions and can't charge in rainy days or at night;

The algorithm parameters and variables used in this paper are defined as follows:

$N_{h}$ - the number of the cluster head nodes needed in the network;

$N$-the number of solar-powered nodes and battery powered nodes;

$N_{s}$ - the number of solar-powered nodes;

$N_{b}$ - the number of battery powered nodes;

$N_{b h}$ - the number of battery powered nodes selected as cluster head nodes;

$N_{\text {shigh }}$ - the number of quasi-cluster head Solar-powered node;

$N_{\text {slow }}$ - the number of downgrade solar-powered node;

Where $N=N_{s}+N_{b}$ and $N_{s}=N_{\text {shigh }}+N_{\text {slow }}$;

$p_{s}$ - The percentage of solar powered nodes with total nodes:

$p_{s}=N_{s} / N=\left(N_{\text {shigh }}+N_{\text {slow }}\right) / N$;

$E s_{i}$ - The residual capacity of solar powered node $i$ is denoted by;

$E b_{j}$ - The residual capacity of battery powered nodes $\mathrm{j}$ is denoted by;
$E_{\text {sthr }}$ - The solar cell has a fixed threshold.

\subsection{Node Classification}

Because Nodes with different power supply types have different energy changing characteristics, all nodes in the network are divided into three types according to their attributes:

Quasi-cluster head solar powered node-Es $\geq E_{s t h r}$. Solar-powered node can get enough energy from the environment in sunny weather. When the solar battery power exceed the fixed threshold $E_{s t h r}$, this solar-powered node is set to be quasi-cluster head Solar-powered node.

Downgrade solar powered node- $E s_{i}<E_{s t h r}$. Solarpowered node can't be charged in raining weather, cloudy weather or at light. When the solar battery power is below the threshold $E_{s t h r}$, this solar-powered node is set to be downgraded solar-powered node.

Battery node-battery powered node gives stable energy supply but can't get energy from the environment. Its energy decreases monotonically.

\subsection{Cluster Head Nodes Selection}

1) Select cluster head node from quasi-cluster head solarpowered nodes

If $N_{\text {shigh }} \geq N_{h}$, that means the number of quasi-cluster head solar-powered node is larger than the number of cluster head node, then select all the cluster head nodes from the solar powered nodes. Each quasi-cluster head solar-powered node has the same probability of being selected as cluster head node. Each quasi-cluster head solar-powered node generates a random number between 0 and 1 . If a node has a random number larger than $T_{1}(n)$, then this node is selected as cluster head nodes. Where

$T_{1}(n)=\frac{N_{h}}{N_{\text {shigh }}}$

2) Select cluster head node from all the nodes

If $N_{\text {shigh }}<N_{h}$, that means in addition to quasi-cluster head solar-powered node, we have to select some cluster head nodes from other nodes.

First we set all the quasi-cluster head solar-powered nodes as cluster head nodes. The probability of being selected to be the cluster head node for each quasi-cluster head solar-powered node is 1.After that, we choose the other $N_{b h}$ cluster head nodes from the battery powered nodes. Where

$N_{b h}=N_{h}-N_{\text {shigh }}$.

The proportion of the battery powered nodes selected as cluster head is $p_{b}=N_{b h} / N_{b}$. 


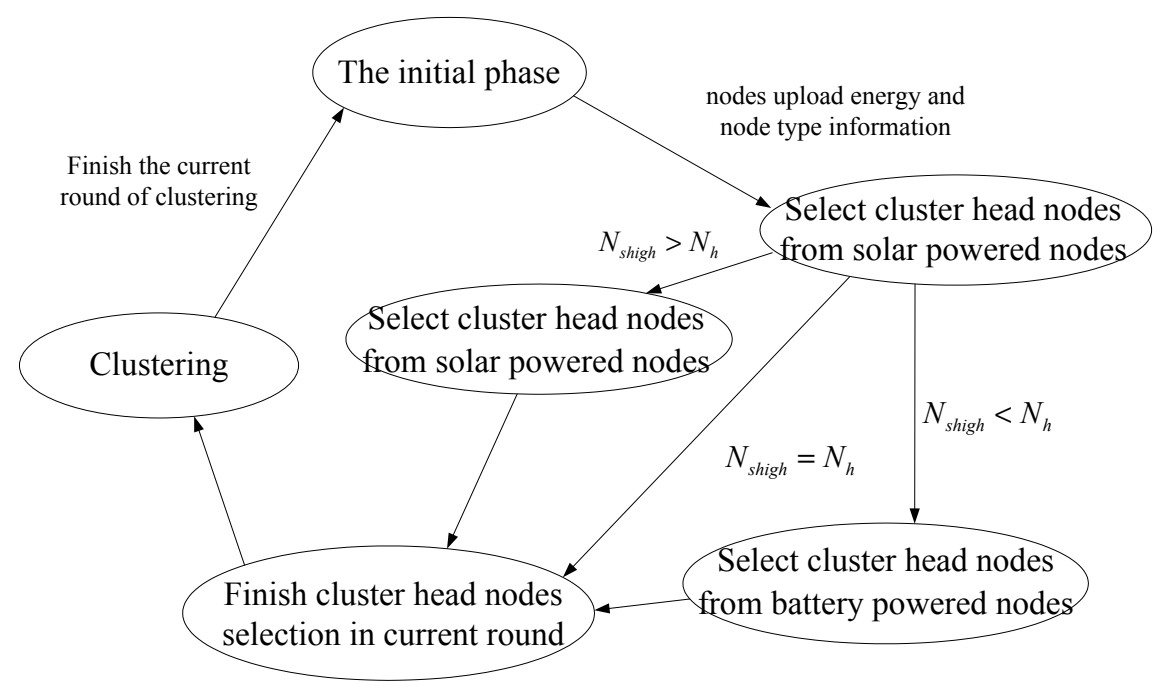

Fig. (2). Clustering process diagram.

Each battery powered node generates a random number between 0 and 1 . If a node has the random number larger than $T_{2}(n)$, then this node is selected as cluster head node.

$T_{2}(n)= \begin{cases}\frac{p_{b}}{1-p_{b} \times\left[r \bmod \left(1 / p_{b}\right)\right]} & , \text { if } n \in G \\ 0 & \text {, therwise }\end{cases}$

Where $p_{b}$ is the percentage of battery-powered nodes selected to be the cluster head node, $r$ is the current round number. $\mathrm{G}$ is the collection of battery powered nodes which are not selected as cluster head nodes in recent $1 / p_{b}$ rounds.

\subsection{Clustering Algorithm Description}

Step 1: nodes characteristic collection

First each node up loads its characteristic message to the sink node. The nodes residual energy information and its power supply type information are included in the characteristics message.

Step 2: nodes classification

After all the characteristic messages have been uploaded, the sink node classifies all the nodes into three types based on the information: Quasi-cluster head Solar-powered node, Downgrade solar powered node and Battery node. The classification process is in accordance with section 4.1. That is, for any node $i$,

If node $i$ is a solar powered node

If the residual energy of node $i \geq E_{\text {sthr }}$

Node $i$ marked as "Quasi-cluster head Solar-powered node"

End if

Else if the residual energy of node $i<E_{\text {sthr }}$

Node $i$ marked as "Downgrade solar-powered node"

End if

End if
Step 3: cluster head competition:

In the cluster head nodes competition stage, the sink node select cluster head nodes in accordance with section 4.2.

$$
\text { If } N_{\text {shigh }}=N_{h}
$$

All the quasi-cluster head solar-powered nodes are marked as cluster head nodes;

$$
\text { Else if } N_{\text {shigh }}>N_{h}
$$

Select cluster head nodes from quasi-cluster head solarpowered nodes.

$$
\text { Else // } N_{\text {shigh }}<N_{h}
$$

All the quasi-cluster head solar-powered nodes are marked as cluster head nodes; choose the insufficient nodes from battery powered nodes.

\section{End if}

Step 4: clustering:

In the clustering stage, the cluster head nodes broadcast CLUSTER_HEAD messages. When the other nodes receives the messages from the cluster head nodes, it chooses a nearest cluster head node as its cluster head node and it depends on the received signal strength and sends a request to this cluster head node to join this cluster. The cluster head receives messages from non-cluster head nodes and sends confirmation message to intra-cluster nodes.

The Fig. (2) is the clustering process diagram.

\section{ANALYSIS}

\subsection{Experimental Scheme}

The software Matlab is adopted in this paper to build a simulation scene and to do performance assessment of this improved algorithm. The simulation scene is a square of size $100 \mathrm{~m} \times 100 \mathrm{~m}$. The total number of nodes a100, of which 20 solar powered nodes and 80batterypowered nodes. The sink 
Table 1. Simulation parameters.

\begin{tabular}{|c|c|}
\hline Parameters & Value \\
\hline \hline The initial energy of battery powered nodes & $2 \mathrm{~J}$ \\
\hline The highest energy of solar powered nodes & $2.5 \mathrm{~J}$ \\
\hline Data packet size & $1000 \mathrm{bit}$ \\
\hline Channel bandwidth & $1 \mathrm{Mbps}$ \\
\hline Node transmission radius & $20 \mathrm{~m}$ \\
\hline$E_{e l e}$ & $50 \mathrm{~nJ} / \mathrm{bit}$ \\
\hline$\varepsilon_{f s}$ & $10 \mathrm{pJ} / \mathrm{bit} / \mathrm{m} 2$ \\
\hline$\varepsilon_{m p}$ & $0.013 \mathrm{pJ} / \mathrm{bit} / \mathrm{m} 4$ \\
\hline$E_{s t h r}$ & $2 \mathrm{~J}$ \\
\hline
\end{tabular}

node is in the center of the square. All the nodes are distributed in the square randomly. The simulation parameters are shown in Table 1.

The solar powered nodes have two states: charge state and discharge state. Charge state corresponds with sunny weather and discharge corresponds with cloudy rainy weather or light.

In the simulation process, charge state and discharge state appear alternately to simulate natural daylight changes. We set one thousand rounds to represent a circadian. We also set a "rainy day" in the simulation process - the solar powered nodes discharge between the 2000th and the 3000th round because of the "rainy day". The simulation shows the performance of the two algorithms in 5000 rounds.

\subsection{Comparative Analysis}

In this section, we compared the performance of the algorithm described in this paper and the LEACH algorithm. The average residual energy of solar powered nodes, the average residual energy of battery powered nodes and the number of surviving node are introduced to show the performance of these two algorithms.

Fig. (3) shows the simulation results:

The Fig. (3a) shows the average residual energy of battery powered nodes using the two algorithms varies with the increase of the round. The figure shows that after several rounds, the network using algorithm described in this paper has a larger average battery residual energy than that of using LEACH algorithm. The network using the improved algorithm consumes more battery energy in the rainy day.

The Fig. (3b) shows the average residual energy of solar powered nodes using the two algorithms varies with the increase of the round. From the figure we can find that the improved algorithm uses more solar energy than the LEACH algorithm. That means using the new algorithm has improved the efficiency of the solar powered nodes, and the network can get more solar energy by using the new energy. And in the rainy days, the improved algorithm uses less solar

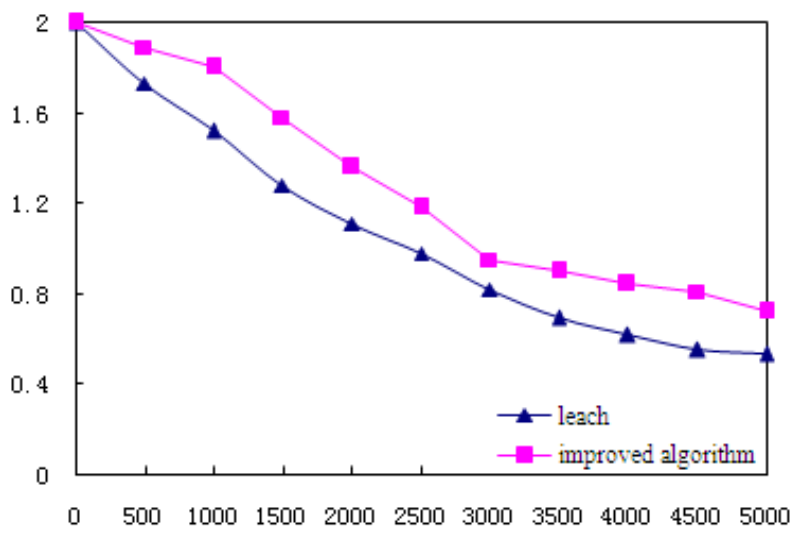

(a) the average residual energy of battery powered nodes

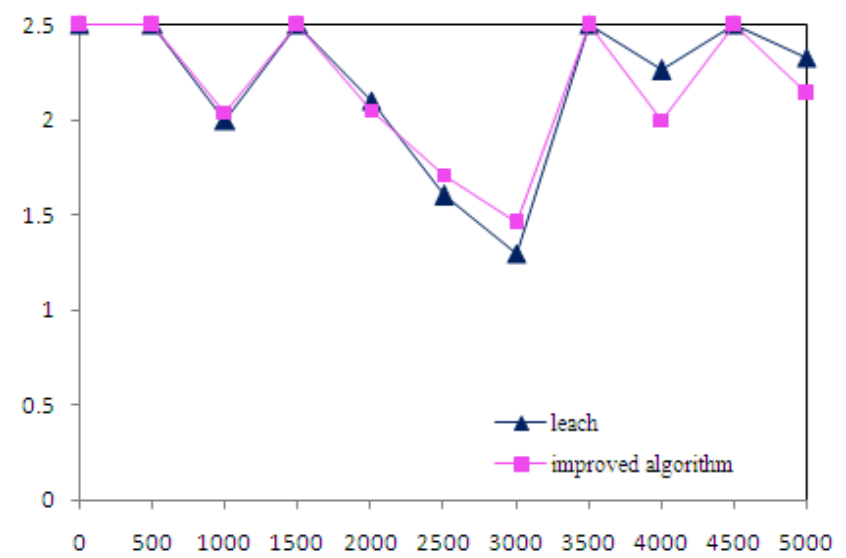

(b) the average residual energy of solar powered nodes

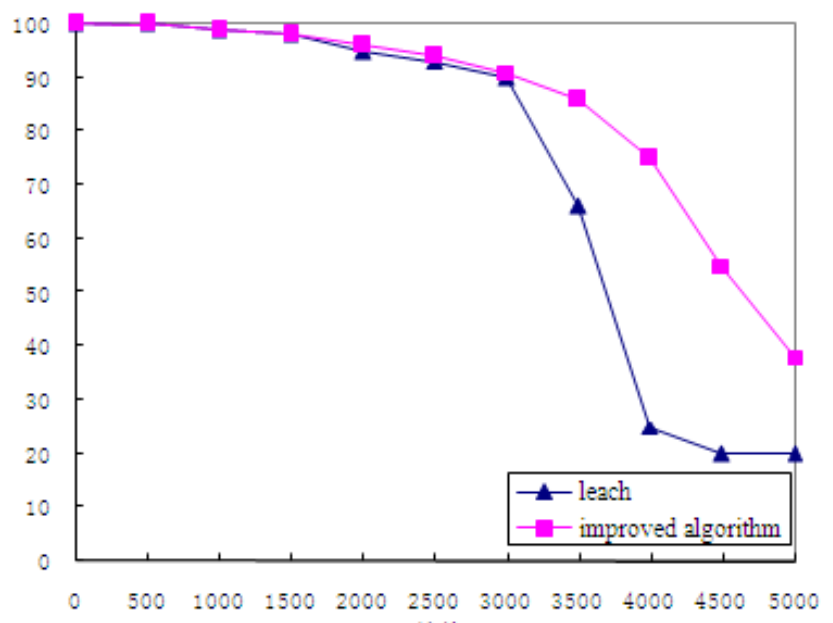

(c) the number of surviving nodes

Fig. (3). The simulate on results.

energy. At the same time, the new algorithm has a larger number of the surviving nodes, See Fig. (3c).

\section{CONCLUSION}

Wireless sensor network use in farmland employs both solar-powered nodes and battery powered nodes can reduce cost and prolong network lifetime. This paper proposed a 
clustering algorithm based on the LEACH algorithm to adapt to this situation. This algorithm first classifies all nodes into 3 types then selects cluster head nodes twice in one round to improve the efficiency of solar node. The simulation results show that this method can improve the utilization of solar energy, reduce the number of deaths node and at last prolong the network life time. Mean while, there are some limitations of this algorithm. If there is a large monitoring area, in which there are no solar powered nodes, it is possible that there are no cluster head nodes in this area; and the weather condition and the work mechanism of the solar powered node are simplified in the simulation process. These questions need be further studied.

\section{CONFLICT OF INTEREST}

The author confirms that this article content has no conflict of interest.

\section{ACKNOWLEDGEMENTS}

This work was supported by Natural Science Foundation of China (61271257); Beijing Municipal Natural Science Foundation (4122034); Natural Science Foundation of China (61102126).

\section{REFERENCES}

[1] M. A. Mirza, R. M. Garimella, "Power aware sectoring based clustering algorithm for wireless sensor networks:'ProcofIntematinal Conference on Information Networking, pp. 1-6, 2009.

[2] J. Edward, "An energy efficient hierarchical clustering algorithm for wireless sensor networks," Proceedings of the IEEE Wireless Communications and Networking Conference, New Orleans, LA, USA: IEEE, pp. 1713-1723, 2003.

[3] L. Shaoqin, "The research of energy efficiency about wireless sensor networks with hierarchy," Shanghai Jiao Tong University, 2012.
[4] Z. Wei-hua, L. La-yuan, Z. Liu-min, and W.Xuan-zheng, " Energy consumption balance improvement of LEACH," Chineese Journal of Computers, vol. 21, no. 11, pp. 1918-1922, 2008.

[5] C. Hai-Bin, J. Xiao-Ming, C. Q. Ying, "Energy prediction and reliable clustering routing protocol for multi level energy heterogeneous wireless sensor networks," Chineese Journal of Computers, vol. 32, no. 12, pp. 2393-2402, 2009.

[6] J. IIbriq, and I. Mahgoub, "Cluster-based routing in wireless sensor networks", Issues and Challenges, vol. 3, no. 4, pp.759-767, 2003.

[7] C. Lei, "Research on algorithms for topology control in heterogeneous wireless sensor networks," 2011.

[8] W. R. Heinzelman, A. Chandrakasan, and H. Balakrishnan, “ Energy-efficient communication protocol for wireless microsensor networks," Proceedings of the Hawaii International Conference on System Sciences, January 4-7, 2000, Maui, Hawaii.

[9] D. K. Noh, and J. Hur, "Energy-aware topology control for reliable data delivery in solar-powered," Journal on Wireless Communications and Networking, vol. 3, p. 258, 2013.

[10] J. Islam, M. Islam, and N. Islam, "A-sLEACH: an advanced solar aware leach protocol for energy efficient routing in wireless sensor networks" Networking, sixth international conference. vol. 2, no. 5, 2007.

[11] L. Huang, and M. J. Neely, "Utility optimal scheduling in energy harvesting networks," IEEE/ACM Translation on Networking, vol. 21, no. 3, pp. 831-844, 2013, May 2011

[12] K, Kinoshita, T.Okazaki, H.Tode and K. Murakami, "A data gathering scheme for environmental energy-based wireless sensor networks," Consumer Communications and Networking Conference, pp. $719-723,2008$

[13] M. Y. Naderi, S. Basagni, and K. R. Chowdhury, "Modeling the residual energy and lifetime of energy harvesting sensor nodes," IEEE GLOBECOM, Anaheim, California, USA, December 3-7, 2012

[14] M. Noori and M. Ardakani, "Lifetime analysis of random eventdriven clustered wireless sensor networks," IEEE Transactions on Mobile Computing, vol. 10, no. 10, pp. 1448-1458, October 2011.

[15] Y. J. Yong, J. Bing, and W. Jie, "An improved algorithm for LEACH protocol in wireless sensor network," Journal of Beijing University of Posts and Telecommunications, vol. 36, no. 1, pp. 105-109, 2013.

[16] C. Xiaojuan, W. Zhuo, and W. Jie, "The improved wireless sensor network routing algorithm based on leach", Chineese Journal of Sensors and Actutors, vol. 26, no.1, pp. 116-121, 2013.

[17] Z. Chen, and G.-X. Lium, "An improved LEACH algorithm based on mobile sensor nodes," CAAI Transactions on Intelligent Systems," vol. 3, no. 2, pp. 140-144, 2008.

Received: September 22, 2014

Revised: November 03, 2014

Accepted: November 06, 2014

(C) Liu et al.; Licensee Bentham Open.

This is an open access article licensed under the terms of the Creative Commons Attribution Non-Commercial License (http://creativecommons.org/licenses/by-nc/3.0/) which permits unrestricted, non-commercial use, distribution and reproduction in any medium, provided the work is properly cited. 